\title{
An Accurate and Fast WLAN User Location Estimation Method Based on Received Signal Strength
}

\author{
Minghua Zhang and Shensheng Zhang \\ Department of Computer Science and Engineering, \\ Shanghai Jiao Tong University, China \\ \{zhangmh, sszhang\} @sjtu.edu.cn
}

\begin{abstract}
Location estimation is a crucial component of location-aware applications. Positioning based on received signal strength (RSS) in wireless networks is considered a promising and inexpensive solution. Existing techniques only use RSS from some fixed access points (APs) deployed within the area of interest to estimate user location. Through experiments on the properties of RSS, it is found that RSS from far access points can distinguish different locations more easily. In this paper, we propose to introduce RSS from APs outside of the area to increase location estimation accuracy. We also present an online maximum matching method to select the most possible locations first, thus reducing the computational cost incurred by using more RSS values and improving the speed of location estimation. Our new location estimation method is implemented and compared with related work in a practical wireless network. Experimental results illustrate that the proposed method can give a higher degree of accuracy.
\end{abstract}

Keywords: User location estimation; Received signal strength; Wireless local area network; Maximum matching; Location estimation speed.

\section{Introduction}

Location estimation is an important task in today's pervasive computing applications [1] that use location information on objects or human beings to provide services without user intervention. A typical positioning system is the Global Positioning System (GPS) [2], yet it doesn't work well in indoor environments or urban areas where high buildings shield the satellite signals. As the proliferation of wireless local area network (WLAN) and the declining cost of mobile devices, great attention have been paid to the received signal strength (RSS)-based location estimation techniques. Such positioning systems build on an already existing, widely available wireless network infrastructure and work indoors as well as outdoors.

RSS-based positioning techniques usually work in two phases: radio map building phase and location determination phase. During the first phase, the received signal strength from observed access points (APs) together with the corresponding sampling position are saved, resulting in a so-called radio map. During the location determination phase, the real-time signal strength measurements are compared to data in the radio map and the nearest match is returned as the estimated user location. In 
indoor environments, the propagation of radio signals suffers from severe multi-path effects, noise and interference [3]. RSS-based location estimation techniques need to deal with the noisy characteristics of the wireless channel. Those characteristics cause the real-time signal strength samples measured in the location determination phase to deviate significantly from those stored in the radio map, thereby limiting the accuracy of such systems.

Current RSS-based techniques can be broadly divided into two categories: deterministic techniques $[4,5,6]$ and probabilistic techniques $[7,8,9]$. Our work lies in the first category. However, none of the previous positioning systems take into account the role of access points outside the area of interest. The RADAR system [4] uses the k-NNSS (k-nearest neighbor in signal space) algorithm. They collect signals from three fixed access points which can be observed in all predetermined locations. The Horus [8] system improves upon the performance of RADAR by using their Joint Clustering technique. Roos [7] adopts RSS from 10 access points deployed within their testbed. In [10], Kaemarungsi investigated the properties of received signal strength reported by IEEE 802.11 b wireless network interface cards. His experimental results indicate that the RSS values from the same AP at two different locations may be difficult to distinguish for positioning purposes when the RSS level is high in which case it tends to have a large degree of variation. To distinguish locations efficiently and improve location estimation accuracy, this finding inspires us to use RSS from the access points deployed not only within the area of interest but also outside of the area. We also propose an online maximum matching method in which the set of observed APs is used to choose possible locations first, alleviating the burden of computation incurred by adopting more RSS values. We test our new location estimation method in a practical wireless network and compare with other work. Experimental results show that the method can achieve a higher degree of accuracy.

The rest of the paper is constructed as follows. Section 2 illustrates the fluctuation of the radio signal strength in WLAN, which is affected by many disturbances. In section 3, we elaborate on our location estimation methodology including the online maximum matching method. We perform experiments and compare our method with others in section 4 . Section 5 concludes the paper and describes some potential future work.

\section{Propagation of Radio Signals in WLAN}

The propagation of radio signals is affected by many disturbances [11]. The IEEE $802.11 \mathrm{~b}$ standard uses license-free $2.4 \mathrm{GHz}$ band to communicate with each other such that other devices using the same band like Bluetooth devices, microwave ovens will be a source of interference. Moreover, $70 \%$ part of a human body is water, and the $2.4 \mathrm{GHz}$ is the resonance frequency of water. Thus human body is an absorber of radio signals.

Multi-path fading [12, 13] is another common phenomenon in radio signal propagation. A transmitted signal can reach the receiver through different paths, each having its own amplitude and phase. These different components combine and produce a distorted version of the transmitted signal. 
Fig. 1 gives a typical example of the normalized histogram of the signal strength received from an access point at a fixed location. People walk in the area, and doors open and close. Such changes in the environment can explain the fluctuation of signal strength shown in the figure.

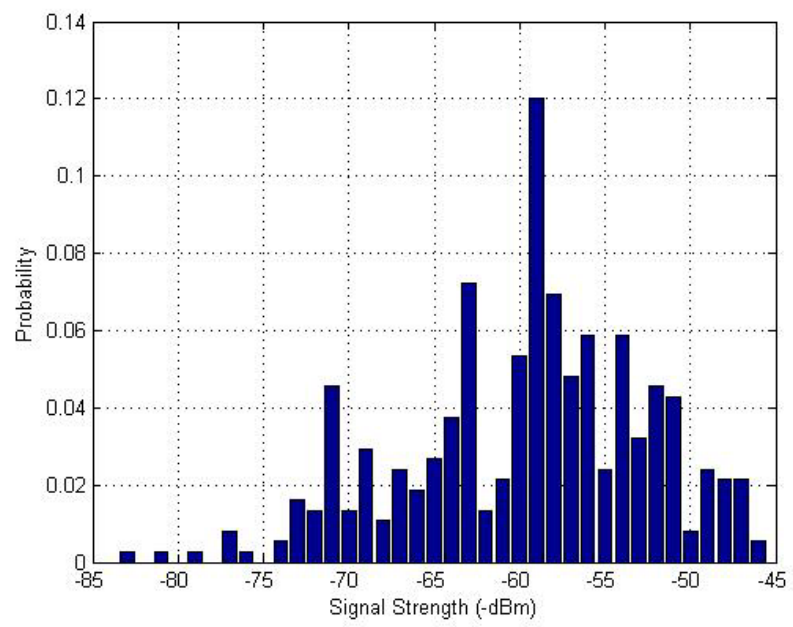

Fig. 1. An example of normalized signal strength distribution

\section{Proposed Location Estimation Method}

\subsection{Radio Map Building Phase}

Suppose the location area $A$ has $\mathrm{N}$ selected sampling positions, $A=\left\{\right.$ position $_{i} \mid 1 \leq \mathrm{i} \leq$ N\}. A RSS sample in our experiment is a 6-tuple: (Id, Time, RSS, SSID, BSSID, Position), whose elements are explained in detail in Table 1.

Table 1. Explanation of a RSS sample vector

\begin{tabular}{|l|l|l|}
\hline Element name & Explanation & Example \\
\hline Id & Sequence number of detected access point & $1,2,3, \ldots$ \\
\hline Time & Sampling date & $2006-05-23$ 18:43:03 \\
\hline RSS & Received signal strength in dBm & $-76,-81, \ldots$ \\
\hline SSID & Service Set Identifier & 1 x.net.sjtu \\
\hline BSSID & MAC address of the access point & $00-13-C 3-59-14-72$ \\
\hline Position & $\begin{array}{l}\text { 2-dimension coordinates and orientation of the } \\
\text { sampling location }\end{array}$ & \\
\hline
\end{tabular}

In each sampling position position $_{i}$, several RSS samples are collected in order to represent the fluctuation of radio signal strength. Then the average value of signal strength $\operatorname{avgrss}_{i j}$ received from each observed AP, whose MAC address is $b s s i d_{j}$, is computed. With the information the radio map is built up. Records in the radio map 
can be described in (1), where L is the number of observed APs in each sampling position.

$$
\begin{aligned}
& r=\left(\text { position }_{i}, \text { avgrss }_{i j}, \text { bssid }_{j}\right), \\
& 1 \leq i \leq N, \\
& 1 \leq j \leq L .
\end{aligned}
$$

\subsection{Location Determination Phase}

During the location determination phase, suppose the real-time RSS samples are represented by (2), where L' is the number of access points detected by user.

$$
U=\left\{\left(m_{-} \text {avgrss }_{i}, m_{-} \text {bssid }_{i}\right) \mid 1 \leq i \leq L^{\prime}\right\}
$$

Before search the nearest location in the radio map, we use an online maximum matching method to choose a subset of radio map containing the possible locations first. As each AP has its own signal's coverage area, the set of observed APs are not totally the same for different locations. Based on the APs in the real-time RSS samples, the positions in the radio map associated with a maximum intersection of the same APs form the sub set $P^{\prime}$. It can be described formally as:

$$
\begin{aligned}
& P^{\prime}=\left\{\text { position }_{i} \mid\left(\forall \text { position }_{r}\right)\left(\left|A P_{m} \cap A P_{i}\right|>\left|A P_{m} \cap A P_{r}\right|\right)\right. \\
& \left.\wedge 1 \leq i \leq N \wedge\left(\text { position }_{r} \in A-P^{\prime}\right)\right\}, \\
& A P_{m}=\left\{U_{n} \cdot m_{-} \text {bssid } \mid 1 \leq n \leq L^{\prime}\right\}, \\
& A P_{i}=\left\{r_{x y} \cdot \text { bssid } \mid r_{x y} \cdot \text { position }=\text { position }_{i} \wedge 1 \leq y \leq L\right\}, \\
& A P_{r}=\left\{r_{x y} \cdot \text { bssid } \mid r_{x y} \cdot \text { position = } \text { position }_{r} \wedge 1 \leq y \leq L\right\} .
\end{aligned}
$$

Then the simple and efficient Euclidean distance is selected as matching metric between real-time RSS samples and RSS data saved in the radio map. The position in $P^{\prime}$, which has a minimum Euclidean distance in signal space is considered as the estimated user location. Euclidean distance is computed by (4):

$$
\begin{aligned}
& \min \left(D_{j}=\sqrt{\sum_{i=1}^{L^{\prime}}\left(U_{i} \cdot m_{-} \text {avgrss }-r_{i j} \cdot{\text { avgrss })^{2}}^{2},\right.}\right. \\
& U_{i} \cdot m_{-} \text {bssid }=r_{i j} \cdot \text { bssid }, \\
& r_{i j} \cdot \text { position } \in P^{\prime} .
\end{aligned}
$$

\section{Experimental Results and Analysis}

\subsection{Experimental Testbed}

We perform our experiments in the second floor of the third building of the Electronic Information and Electrical Engineering School buildings. The layout of the floor is 
shown in Fig. 2. The area has a dimension of 100 meters by 20 meters. It includes a long hallway, a meeting room, lots of offices and labs.

To measure the RSS from different APs, we use an ASUS pocket PC with an embedded wireless Ethernet card to capture the signal. We use WRAPI [14], a third party library for retrieving the signal strength.

There are altogether 30 access points that can be detected. They are deployed not only in the second floor, but also in other floors or in neighboring buildings. For building up the radio map, we take the sampling locations along the hallway on a grid with cells of $5 \times 2$ meters (the hallway width is 2 meters). For each location, 10 samples are collected for each orientation.

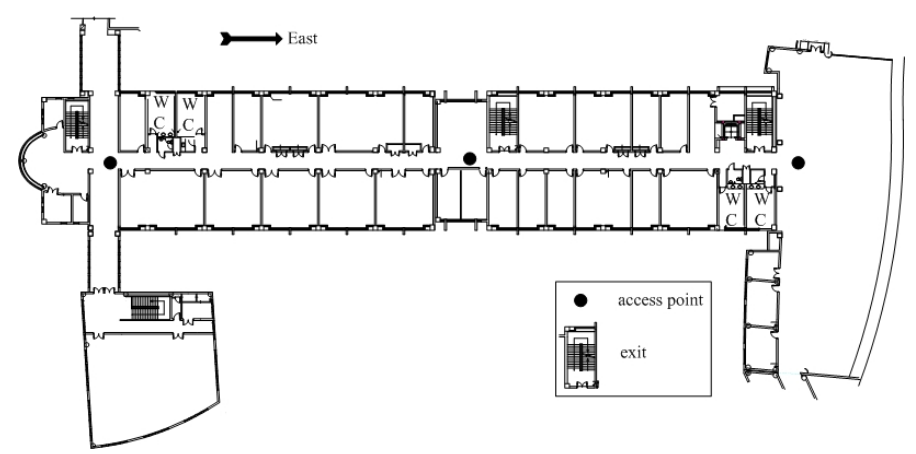

Fig. 2. The layout of the second floor of the third building of the Electronic Information and Electrical Engineering School buildings

\subsection{Experimental Results}

In this section, we evaluate the performance of our proposed method. An independent test set collected on different time and day from that for the radio map building phase is used. We conduct experiments and compare with other methods. In the first experiment, we only use the RSS values from access points deployed within the testbed. The method is just like the 1-NNSS method in the RADAR system, so we call it the RADAR method in Fig. 3. In the second experiment, we only adopt the RSS data from three strongest APs in each sampling position, which is called as the 3strongest AP method. This method is similar to the technique in [8] on the selection of RSS data. In the last experiment, we use RSS data from all observed APs.

Experimental results are illustrated in Fig. 3. Location estimation error is defined as the Euclidean distance between the real position and its estimated position. The results of our proposed method are quite positive, for it is more accurate than the other two methods. Fig. 4 gives the cumulative distribution of error distance in the three experiments. Using the proposed method, over 73 percent positions are located within 5 meters, which is higher than the other two methods. This demonstrates that more RSS data can improve the location estimation accuracy. 


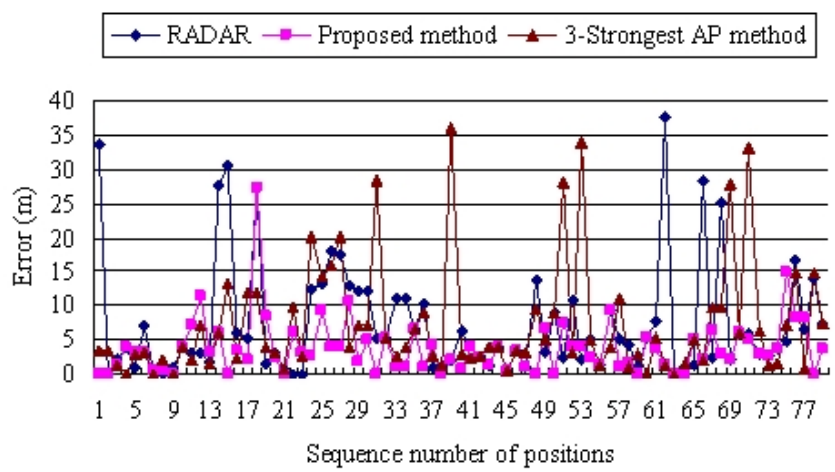

Fig. 3. Comparison of location estimation error in the three experiments

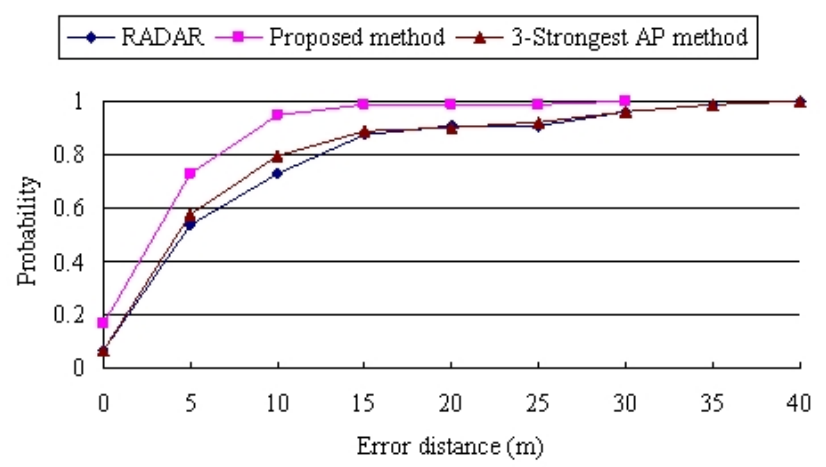

Fig. 4. Error distance CDF of the three experiments

Since we use RSS from more access points to estimate user location, it is possible that the calculating time will increase. Therefore we also perform an experiment to measure the locating time. The personal computer is configured with $512 \mathrm{MB}$ of RAM, Intel(R) Pentium(R) M processor $1.5 \mathrm{GHz}$. The three methods are implemented in MATLAB and Table 2 lists the CPU time each method uses. In general, the more access points' signal strength information is used, the more locating time is needed. Although the number of access points used in our method is much more than the RADAR method, yet the difference of CPU time needed is very small ( 0.13 seconds). However the average error of the RADAR method is about two times that of our proposed method. Therefore it is a tolerable compromise for the improvement of location estimation accuracy. In the Table 2, the tremendous increase of CPU time spent on the 3-strongest AP method is due to the sort operation to choose the first three strongest access points. On the location estimation accuracy, it is very close to the RADAR method. 
Table 2. Compare locating time of the three experiments

\begin{tabular}{|l|l|l|l|}
\hline Comparison Items & Proposed Method & RADAR & 3-Strongest AP Method \\
\hline Number of access points matched & 15 & 3 & 3 \\
\hline Average error $(\mathrm{m})$ & 3.74 & 7.43 & 7.29 \\
\hline CPU time (s) & 0.86 & 0.73 & 1.15 \\
\hline
\end{tabular}

\section{Conclusion and Future Work}

Mobile user's current location is one of the most important and frequently-used contexts in pervasive computing environments. Positioning based on received signal strength is considered a promising and inexpensive solution. However, received signal strength especially in indoor environments is interfered by many disturbances. This paper proposes a new user location estimation method to improve accuracy, which uses RSS data from access points deployed not only within the area of interest but also outside of the area. RSS data from the far APs are used to better distinguish locations. Moreover an online maximum matching method is presented to quickly get the most possible locations in order to improve the speed of location estimation.

Experimental results show that, with the $2 \times 5$ meters sampling grid, our method can estimate user location to within 5 meters with over $73 \%$ accuracy. In the same testbed, the proposed method is better than the methods used in other work on accuracy. It is also remarkable that the sampling grid in the proposed method is bigger than that in the related work, which means fewer calibration efforts are needed. Furthermore, through another experiment the increase of locating time by using more access points is negligible relative to the improvement of location estimation accuracy.

In this paper, the proposed method belongs to the deterministic technique. But the idea of using more RSS data from far APs and the online maximum matching method are not limited to it. They can also be used in the probabilistic technique. In the future, we would like to do more extensive experiments, studying the effect of parameters such as the size of sampling grid, the number of access points and try to prove our idea in a formal way.

Acknowledgments. This research is supported by Shanghai Commission of Science and Technology Grant (05SN07114, 03DZ19320). The authors would like to thank Haibin Mei for his valuable advice. Thanks also to anonymous reviewers for their perspicacious comments.

\section{References}

1. Hazas, M., Scott, J., Krumm, J.: Location-Aware Computing Comes of Age. IEEE Computer, Vol. 37, No. 2, (2004) 95-97.

2. Enge, P., Misra, P.: Special Issue on GPS: The Global Positioning System. Proceedings of the IEEE, Vol. 87, No. 1, January (1999) 3-172.

3. Hashemi, H.: The Indoor Radio Propagation Channel. Proceedings of the IEEE, Vol. 81, No. 7, (1993) 943-968. 
4. Bahl, P., Padmanabhan, V. N.: RADAR: An In-building RF-based Location and Tracking System. In: Proceedings of the IEEE INFOCOM 2000, Tel-Aviv Israel, March (2000) 775-784.

5. Ahmad, U., Gavrilov, A., Sungyoung Lee, Young-Koo Lee: Modular Multilayer Perceptron for WLAN Based Localization. In: Proceedings of 2006 International Joint Conference on Neural Networks, Vancouver BC, Canada, July (2006) 3465-3471.

6. Smailagic, A., Kogan, D.: Location Sensing and Privacy in a Context-Aware Computing Environment. IEEE Wireless Communications, Vol. 9, No. 5, October (2002) 10-17.

7. Roos, T., Myllymaki, P., Tirri, H., Misikangas, P., Sievanen, J.: A Probabilistic Approach to WLAN User Location Estimation. International Journal of Wireless Information Networks, Vol. 9, No. 3, July (2002) 155-164.

8. Youssef, M., Agrawala, A., Shankar, A. U.: WLAN Location Determination via Clustering and Probability Distributions. In: Proceedings of IEEE International Conference on Pervasive Computing and Communications. IEEE Computer Society (2003) 23-26.

9. Castro, P., Chiu, P., Kremenek, T., Muntz, R.: A Probabilistic Location Service for Wireless Network Environments. In: Proceedings of the International Conference on Ubiquitous Computing. Springer LNCS 2201, Berlin Heidelberg (2001) 18-24.

10. Kaemarungsi, K., Krishnamurthy, P.: Properties of Indoor Received Signal Strength for WLAN Location Fingerprinting. In: Proceedings of the First Annual International Conference on Mobile and Ubiquitous Systems - Networking and Services. IEEE Computer Society (2004) 14-23.

11. Ladd, A. M, et al.: Robotics-Based Location Sensing using Wireless Ethernet. In: Proceedings of the 8th Annual International Conference on Mobile Computing and Networking. ACM Press (2002) 227-238.

12. Stallings, W.: Wireless Communications and Networks. First Edition. Prentice Hall (2002).

13. Youssef, M., Agrawala, A.: Small-Scale Compensation for WLAN Location Determination Systems. In: Proceedings of the IEEE WCNC 2003, Vol. 3. March (2003) 1974-1978.

14. WRAPI. Website: http://ramp.ucsd.edu/pawn/wrapi/. 